\title{
Efeitos do envelhecimento no contraste visual de grades senoidais
}

\author{
Maria José Nunes Gadelha \\ Joenilton Saturnino Cazé da Silva \\ Jandilson Avelino da Silva \\ Michael Jackson de Oliveira Andrade \\ Débora Najda de Medeiros Viana \\ Natanael Antonio dos Santos \\ Universidade Federal da Paraiba - PB, Brasil
}

\begin{abstract}
Resumo
O objetivo desse estudo foi comparar as curvas de sensibilidade ao contraste de adultos e idosos. Foi medida a FSC para grades senoidais verticais utilizando o método psicofísico da escolha forçada (2AFC). Participaram desse estudo 18 adultos com idade entre 20 e 29 anos e 18 idosos com idade entre 60 a 69 anos, de ambos os sexos. A ANOVA mostrou efeito principal entre os grupos $\left[\mathrm{F}_{(1,156)}=173,160 ; \mathrm{p}<0,001\right]$ e de interação entre as frequências espaciais e a idade $\left[\mathrm{F}\left({ }_{3,427)}=163,365 ; \mathrm{p}<0,001\right]\right.$. Já o teste Bonferroni mostrou diferenças significantes em todas as frequências testadas $(\mathrm{p}<0,001)$. Verificou-se que o padrão da curva de $\mathrm{SC}$ se mantém com o envelhecimento, com a menor sensibilidade localizada nos extremos da curva e a maior sensibilidade localizada próxima ao centro da curva. Além disso, sugere-se alterações no processo senso perceptivo visual ao longo da vida.

Palavras-chave: Método psicofísico; Percepção; Contraste visual; Envelhecimento.
\end{abstract}

\section{Effects of aging on visual contrast of sinusoidal gratings}

\begin{abstract}
The aim of this study was to compare the behavior of the contrast sensitivity curve and older adults. We measured the CFS for vertical sinusoidal gratings using the psychophysical forced-choice method (2AFC). Participants were 18 volunteers aged 20-29 years old $(\mathrm{M}=23.94, \mathrm{SD}=2.57)$, and 18 elderly aged 60-69 years old $(\mathrm{M}=66.05, \mathrm{SD}=3.65)$ of both sexes. The ANOVA showed main effects between groups $\left[\mathrm{F}_{(1,156)}=173.160 ; \mathrm{p}<0.001\right]$ and interaction between the frequencies and age $\left[\mathrm{F}_{(3,427)}=163.365\right.$; $\mathrm{p}<0.001]$. But the Bonferroni showed significant differences in all tested frequencies $(\mathrm{p}<0.001)$. It was found that the standard curve CS is maintained with aging, with the lowest sensitivity is located at the extreme bow and greater sensitivity is near the center of the curve. Furthermore, is suggested changes in visual sense perceptual process throughout life.
\end{abstract}

Keywords: Psychophysical method; Perception; Visual contrast; Aging.

\section{Efectos del envejecimiento en el contraste visual de rejillas sinusoidales}

\section{Resumen}

El objetivo de este estudio fue comparar las curvas de sensibilidad al contraste (SC) en adultos y ancianos. La FSC se midió por rejillas sinusoidales verticales utilizando el método psicofísico de elección forzada (2AFC). Los participantes fueron 18 adultos de edades comprendidas entre 20 y 29 años y 18 ancianos de 60 a 69 años, de ambos sexos. La ANOVA mostró diferencias significativas en los efectos principales de los grupos $\left[\mathrm{F}_{(1,156)}=173.160 ; \mathrm{p}<0.001\right]$ y una interacción entre las frecuencias y la edad $\left[\mathrm{F}_{(3,427)}=163.365 ; \mathrm{p}<0,001\right]$. En el test de Bonferroni fueron encontradas diferencias significativas en todas las frecuencias analizadas $(\mathrm{p}<0,001)$. Se encontró que el estándar de la SC se mantiene con el envejecimiento, teniendo la zona de menor sensibilidad situada en el extremo de la curva y una mayor sensibilidad cerca del centro de la curva. Además, se sugiere cambios en el sentido visual y perceptual a lo largo de la vida.

Palabras clave: Método psicofísico; Percepción; Contraste visual; Envejecimiento. 
O envelhecimento é considerado um processo gradual de declínio em estrutura, função, organização e diferenciação, culminando por fim na morte do indivíduo (Malloy-Diniz, Fuentes, \& Cosenza, 2013). As alterações resultantes deste processo acometem diversas estruturas no Sistema Nervoso Central (SNC) e Periférico (SNP), refletindo no funcionamento dos inúmeros mecanismos a eles associados, como no caso dos sensoriais. Os órgãos do sentido, por si só, acabam sofrendo comprometimento, determinando privações sensoriais e perceptivas que contribuem para o declínio cognitivo, notoriamente presente nesta faixa etária (Bishop, Lu, \& Yankner, 2010).

Ao longo dos anos diversas investigações demonstraram que existe um declínio significativo das funções cognitivas e sensoperceptivas com o avanço da idade (Elliott \& Werner, 2010; Li \& Lindenberger, 2002; Mateus et al., 2013; Salthouse \& Czaja, 2000; Silva, d'Almeida, Oliveiros, Mateus, \& CasteloBranco, 2014). O sistema visual mostra-se vulnerável aos efeitos negativos do envelhecimento (Birren \& Schaie, 2006), sendo a funções de detecção de contraste um dos componentes mais afetados pelo avanço da idade (Burton, Owsley, \& Sloane, 1993). A redução desta capacidade visual, juntamente com a acuidade, é considerado um fator de risco para o aumento de quedas e acidentes com veículos para a população desta faixa etária (DeLoss, Watanabe, \& Andersen, 2015).

O sistema visual humano tem a capacidade de detectar e processar estímulos de diversas dimensões e níveis de contraste. A Sensibilidade ao Contraste (SC) possibilita o processamento visual da forma, permitindo a identificação de objetos, sendo definida como a quantidade mínima de contraste necessária para detectar um determinado padrão visual ou frequência espacial (Cornsweet, 1970).

Uma das ferramentas para a mensuração e investigação dos atributos ópticos e neurais da visão é a Função Sensibilidade ao Contraste - FSC (Campbell, 1983). Ela pode ser descrita como uma medida que determina a habilidade do sistema visual em detectar padrões de frequências espaciais diferentes em níveis baixos de contraste. Assim, a FSC se apresenta como o inverso da curva de SC (Santos \& França, 2008).

A relação entre envelhecimento normal e SC foi investigada ao longo dos anos por meio de diversas pesquisa que mostraram haver um declínio da curva deste atributo (Arundale, 1978; McGrath \& Morrison, 1981; Owsley, Sekuler, \& Siemsen, 1983; Skalka, 1980). Outros estudos corroboraram com a hipótese da existência de alterações na FSC, ao longo do envelhecimento humano (Santos, Simas, \& Nogueira,
2003), porém existem discrepâncias quanto a alguns dados da literatura, conforme observado nas revisões de Crassini, Brown e Bowman (1988) e Owsley, Sekuler e Siemsen (1983). Estas discrepâncias nos resultados das pesquisas fazem menção às faixas de frequência que são afetadas pelo processo de envelhecimento, sendo que a variedade de resultados encontrados provavelmente se deve à diversas quantidades de técnicas distintas utilizadas para a mensuração da FSC (Costa et al., 2009). Por exemplo, Burton et al. (1993) relataram uma perda na sensibilidade ao contraste para todas as frequências espaciais (McGrath \& Morrison, 1981; Skalka, 1980), enquanto outros verificaram perdas nas frequências espaciais médias e altas (Arundale, 1978; Derefeldt, Lennerstrand, \& Lundh, 1979; Owsley et al., 1983). Além disso, Sekuler e Hutman (1980) encontraram prejuízos apenas em frequências espaciais baixas e médias.

As alterações encontradas em estruturas ópticas e neurais do sistema visual ainda são sugestivas para buscar o entendimento da curva normal de sensibilidade ao contraste espacial, principalmente quando relacionado a frequências espaciais baixas (Habak \& Faubert, 2000; Higgins, Jaffe, Caruso, \& deMonasterio, 1988; Schefrin, Tregear, Harvey Jr, \& Werner, 1999).

Pesquisas relacionadas a percepção visual mostram que curvas de SC podem ser bastante úteis na detecção de simples padrões visuais em forma de ondas senoidais espaciais com orientação vertical e horizontal (Kalia et al., 2012; Jia, Yan, Hou, Lu \& Huang, 2014; Lesmes, Jackson, Wallis, Bex, 2013; Scialfa, Kline \& Wood, 2002; Skalka, 1980; Pelli \& Bex, 2013). Esses diferentes padrões comportamentais distinguem maneiras dos mecanismos neurais processarem a informação (Costa et al., 2013; Ellemberg, Lewis, Hong Liu, \& Maurer, 1999; Santos, Oliveira, Nogueira, Cruz, \& Simas, 2006). Neste sentido, este estudo teve por objetivo avaliar a curva de SC de adultos e idosos saudáveis para estímulos de grades senoidais verticais utilizando frequências de 0,$6 ; 2,5 ; 5$ e 20 cpg em condições de luminância fotópica.

\section{MÉTODO}

\section{Participantes}

Participaram desse estudo 18 voluntários com idade entre 20 e 29 anos $(M=23,94 ; D P=2,57)$, e 18 idosos com idade de 60 a 69 anos $(M=66,05 ; D P=3,65)$. Os grupos possuíam voluntários de ambos os sexos. Foram requeridos como critérios de inclusão a qualidade de saúde dos participantes e a ausência de doenças e anomalias oculares, como por exemplo, glaucoma, 
catarata, estrabismo e ambliopia. Além disso, os idosos estavam livres de transtornos que pudessem afetar o envelhecimento normal. Todos apresentavam acuidade visual binocular normal ou corrigida (20/20) avaliada por meio da optométrica "E" de Rasquim a uma distância de $600 \mathrm{~cm}$ da cartela. O Mini-Exame do Estado Mental - MMSE (Folstein, Folstein, \& McHugh, 1975) foi aplicado nos participantes idosos como uma medida neuropsicológica e os dados mostraram ausência de prejuízo cognitivo (Bertolucci, Brucki, Campacci, \& Juliano, 1994).

A participação ocorreu de forma voluntária por meio do Termo de Consentimento Livre e Esclarecido, devidamente assinado, de acordo com os parâmetros estabelecidos pela Resolução no 466/12 do Conselho Nacional de Saúde (Ministério da Saúde). O estudo foi aprovado por Comitê de Ética do Hospital Universitário Lauro Wanderley sob protocolo no 229/11.

\section{Equipamento e estímulos}

Foi utilizado como aparato para medir a SC um monitor de vídeo colorido LG/RCT (Cathodic Ray Tube) com tela plana de 19 polegadas. Esse monitor era controlado por um microcomputador com uma placa de vídeo com entradas VGA e DVI, conectadas por um hardware Bits++ (Cambridge Research Systems, Rochester, Kent, England), que tinha por função aumentar a resolução da tela do monitor de 8 para 14 bits, possibilitando a geração de estímulos com melhor definição. Essa tela apresentava resolução de

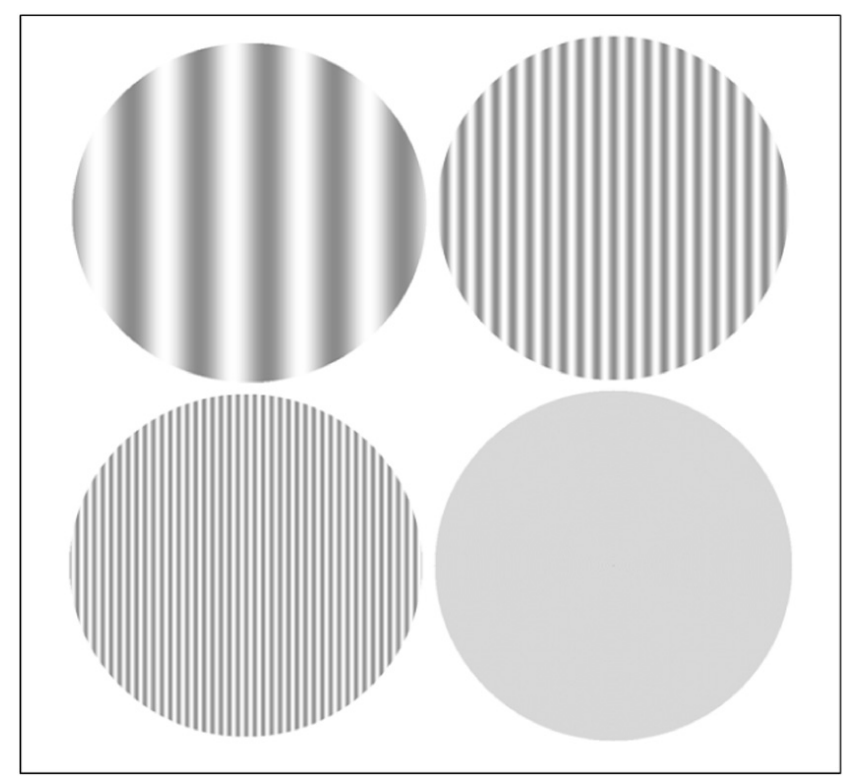

Figura 1. Representação de estímulos de grade senoidal vertical e neutro. Acima: esquerda, 0,6 cpg; direita, 2,5 cpg. Abaixo: esquerda, 5 cpg; direita, estímulo neutro.
$1.024 \times 768$ pixels com taxa de atualização de $70 \mathrm{~Hz}$ e valor de luminância média com $40,1 \mathrm{~cd} / \mathrm{m}^{2}$. A luminância e a correção gama do monitor foram reguladas por um programa LightScan e um fotômetro OptiCAL (Cambridge Research Systems, Rochester, Kent, England).

Utilizaram-se estímulos visuais acromáticos do tipo grade senoidal vertical com frequências espaciais de 0,$6 ; 2,5 ; 5,0$ e 20 ciclos por grau de ângulo visual, e um estímulo neutro cinza homogêneo com luminância média (Figura 1). Todos os estímulos foram gerados no centro da tela do monitor com diâmetro de 7,2 graus de ângulo visual em formato circular. Os estímulos foram gerados com programação de linguagem $\mathrm{C}++$ desenvolvido pelo próprio laboratório onde foi desenvolvida a pesquisa.

O ambiente experimental tinha dimensão de $5 \mathrm{~m}^{2}$ e iluminação de 400 lux. Foi fixado uma cadeira a uma distância de $150 \mathrm{~cm}$ da tela do monitor e fincado um mouse para os participantes realizarem suas respostas a medida que fossem estimulados.

\section{Procedimento}

Empregou-se um delineamento experimental com medidas repetidas, no qual o "N" estatístico foi obtido a partir do número de reversões ou valores de máximos e mínimos de contraste. Utilizaram-se como variáveis independentes, a idade e as frequências espaciais, e como variável dependente, o valor de limiar ao contraste. Todos os participantes passaram pelas mesmas condições em momentos e ordem diferentes. $\mathrm{O}$ efeito de ordem foi controlado pela técnica de contrabalanceamento.

A sensibilidade ao contraste foi medida pelo paradigma psicofísico da escolha forçada entre duas alternativas temporais (2AFC). Este método, utilizado originalmente por Wetherill e Levitt (1965), calcula a probabilidade de acertos consecutivos por parte dos participantes, ou seja, em cerca de 100-150 apresentações do par de estímulos (neutro e teste), o estímulo de teste tinha probabilidade de ser percebido em média $75 \%$ das vezes.

As sessões experimentais de teste e de reteste consistiram na apresentação sucessiva simples de pares de estímulos, sendo um deles uma frequência de teste (com uma das frequências espaciais estudadas) e o outro, um estímulo neutro de padrão homogêneo com luminância média. Adotaram-se, como critério, três acertos consecutivos para diminuir o contraste em $20 \%$, e um erro para aumentar o contraste na mesma unidade. Cada estímulo foi apresentado por $2 \mathrm{~s}$, com intervalo de $1 \mathrm{~s}$ entre eles e de $3 \mathrm{~s}$ entre tentativas, separadas por um sinal sonoro (Figura 2). 


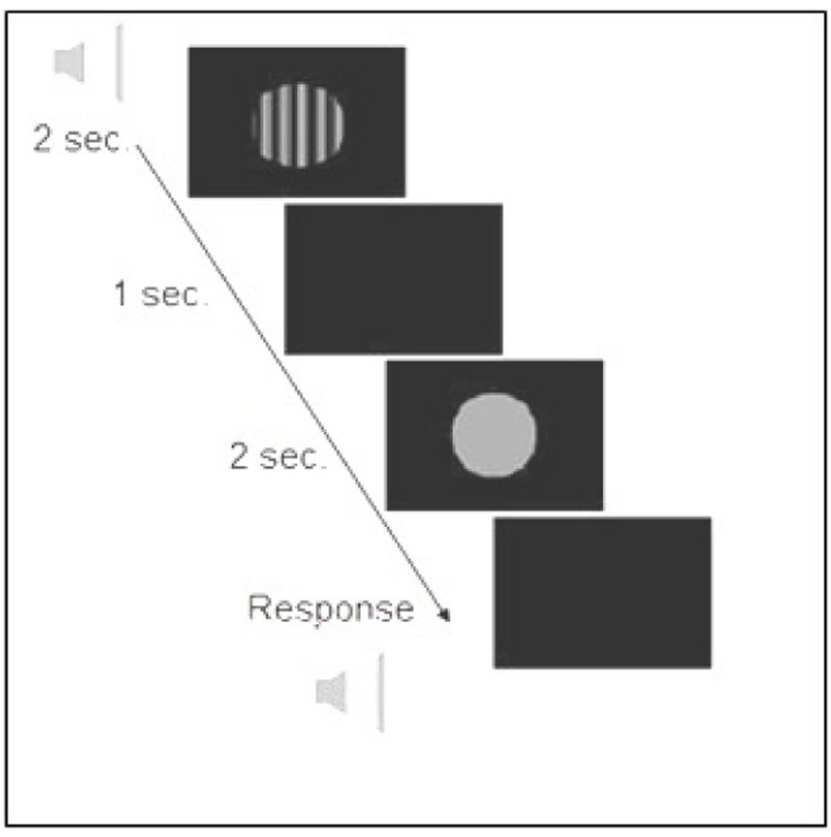

Figura 2. Variação do tempo (em segundos) entre os pares de estímulos durante a sessão experimental.

Antes do início de cada sessão experimental, os voluntários foram orientados a pressionar o botão esquerdo do mouse (botão $\mathrm{n}$ - 1 ) quando o estímulo de teste fosse apresentado primeiro, e o botão direito do mouse (botão $\mathrm{n}^{\mathrm{O}} 2$ ) quando o estímulo de teste fosse apresentado em segundo lugar. Cada sessão foi iniciada com o estímulo teste com contraste em nível supralimiar, e os experimentos só começaram quando o experimentador certificou-se de que os participantes responderiam conforme as instruções elucidadas.

As sessões experimentais tinham duração média de 10 a 15 minutos e cada frequência foi medida duas vezes em diferentes dias. Utilizou-se o critério de uma semana como tempo para realizar entre uma medida e outra, além disso todas as medidas aconteceram no mesmo turno do dia. Nesse tipo de procedimento, o número de apresentações necessárias para determinar o limiar de contraste varia de acordo com os acertos e erros dos voluntários, bem como do número de máximos e mínimos ou reversões que se quer obter. Neste estudo, a sessão experimental terminou automaticamente assim que três valores máximos e três mínimos de contrastes (ou seis reversões) foram obtidos.

\section{Resultados}

Após cada sessão experimental foi gerado um protocolo de resultados contendo 10 valores de limiar de contraste. Porém foram aceitos apenas seis valores de limiares, retirando os dois inicias (critério de entusiasmo) e os dois finais (critério de fadiga). Os valores de máximos e mínimos de contrastes obtidos para cada frequência foram transformados em SC (1/limiar de contraste) e em seguida agrupados em planilhas de acordo com a idade, como adultos jovens e idosos.

A Figura 3 mostra as curvas de SC visual para ambos os grupos para as frequências de 0,$6 ; 2,5 ; 5,0$ e $20 \mathrm{cpg}$. A SC é apresentada no eixo das ordenadas e as frequências espaciais no eixo das abscissas. Os erros padrões das médias para cada frequência espacial são indicados pelas barras verticais.

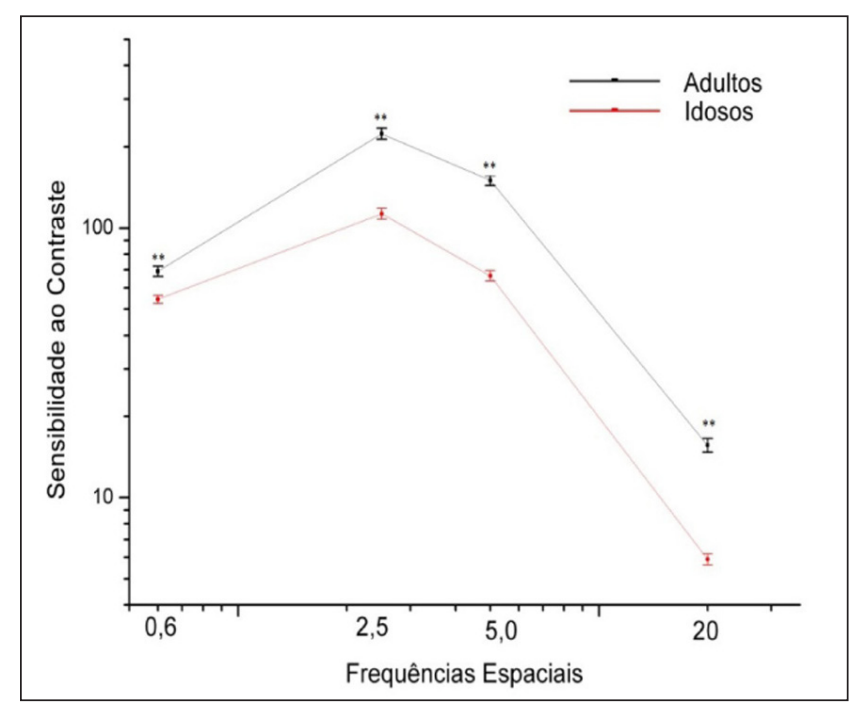

Figura 3. Curvas de sensibilidade ao contraste com frequências espaciais de 0,$6 ; 2,5 ; 5,0$; e $20 \mathrm{cpg}$ para adultos e idosos. $* * \mathrm{p}<0,001$.

A análise de variância Repeated Measures ANOVA mostrou efeito principal entre os grupos $\left[\mathrm{F}_{(1,156)}=173,160 ; p<0,001\right]$ e de interação entre as frequências e a idade $\left[\mathrm{F}_{(3,427)}=163,365 ; p<0,001\right]$. Já o teste post-hoc Bonferroni mostrou diferenças significantes em todas as frequências testadas $(p<0,001)$. Além disso, pode-se verificar que o valor de maior e menor sensibilidade aconteceram nas frequências de 2,5 e $20 \mathrm{cpg}$, respectivamente, tanto para os jovens adultos como para os idosos. Contudo, os jovens adultos obtiveram razões de sensibilidade ao contraste em todas as frequências $0,6(1,3) ; 2,5(2,0)$; $5(2,3)$ e $20(2,7)$ superiores aos dos idosos.

\section{Discussão}

A contradição entre os últimos resultados de pesquisas que relatam sobre o contraste visual espacial levou esse trabalho a tentar esclarecer como 
os canais visuais se comportam durante o processo de envelhecimento. Dessa forma, o objetivo desse estudo foi comparar o comportamento da curva de SC de adultos e idosos saudáveis, isto é, sem patologias identificáveis, para frequências espaciais baixas, médias e altas. Foi verificado que o padrão da curva de SC se mantém com o envelhecimento, com menor sensibilidade localizada nos extremos da curva, em frequências espaciais baixas e altas, e maior sensibilidade localizada próxima ao centro da curva, frequências médias de 2,5 cpg (Figura 3), sendo esse padrão registrado desde a adolescência, e continuando durante a fase adulta (Andrade et al., 2013). Apesar do perfil da SC ter se apresentado semelhante em adultos e idosos, as análises estatísticas mostraram diferenças significantes entre os grupos para todas as frequências $(0,6 ; 2,5 ; 5$ e $20 \mathrm{cpg})$, de forma que os adultos jovens foram mais sensíveis que os idosos.

Esses resultados corroboram com outros dados da literatura, mesmo apresentando métodos diferenciados (Burton et al., 1993; Costa et al., 2013; Costa et al., 2009; McGrath \& Morrison, 1981; Skalka, 1980). Burton et al. (1993) verificou monocularmente a SC de adultos jovens (17-29 anos) e idosos (60-80 anos) por meio do paradigma dos estímulos constantes e percebeu uma diminuição no contraste visual para frequências de $2,4,8,12,16,24,28$ e 32 cpg. Além disso, Santos et al. (2006), utilizando uma amostra de adultos (19-23 anos) e idosos (60-65 anos) em condição mesópica de luminância, também mostrou diferenças significantes no contraste visual para frequências baixas, médias e altas. Esses dados permanecem mesmo utilizando padrões de estímulos espaciais diferentes, ou seja, grades senoidais verticais e concêntricas. Apesar das diferenças terem sido identificadas em todas as frequências, diferenças maiores foram detectadas na frequência espacial média $(2,5 \mathrm{cpg})$ e nas freqüências altas (5,0 e $20 \mathrm{cpg}$ ), em que os adultos foram mais sensíveis que os idosos na razão de 2,0;2,3 e 2,7, respectivamente.

Procurando entender como os mecanismos visuais se comportam ao longo de diferentes faixas etárias, Owsley et al. (1983) avaliaram a acuidade visual, a pressão ocular e a SC (frequências espaciais de 0,5; 1, 2, 4, 8 e 16) em condições de luminância fotópica $\left(103 \mathrm{~cd} / \mathrm{m}^{2}\right)$. Os resultados se mostraram distintos em relação aos anteriores, mesmo utilizando o método de retirada de limiares iniciais, como utilizado no presente estudo. Contudo, a diminuição da SC aconteceu a partir dos 50 anos com pico máximo aos 60 anos, e só foi observado diferença nas frequências espaciais médias e altas. Os autores discutiram que os resultados encontrados foram devido à baixa capacidade de percepção de luz afetada pelo processo de envelhecimento.

Resultados parecidos também foram encontrados por Schefrin et al. (1999). Estes autores mostraram que existe uma redução da SC para grades senoidais horizontais apenas para frequências espaciais altas, conforme também citado por Tang e Zhou (2009). Allard, Renaud, Molinatti e Faubert (2013) apontam que a capacidade de atenuação em frequências altas pode estar relacionada a localização de ruído interno e externo aos estímulos.

Diante dessas evidências pode-se levantar a hipótese de que diferentes mecanismos do sistema visual sejam atingidos pelo processo de envelhecimento. É possível que todas as frequências espaciais sofram alterações ao longo da vida, mas que as frequências médias e altas sejam ainda mais afetadas. Essas reduções são baseadas nas hipóteses de declínio neurobiológico e na capacidade psicofísica de detectar os estímulos, como por exemplo, a relevância dos estímulos e os paradigmas aplicados (Mateus et al., 2013). Além disso, as alterações na curva de SC encontradas em idosos podem ser causadas por perdas ópticas (Bissig, Roberts, \& Berkowitz, 2011; Park et al., 2004; Roorda, 2011) e neurais (Elliott \& Werner, 2010; Lesmes, Wallis, Lu, Jackson, \& Bex, 2012; Rosen et al., 2015) típicas do envelhecimento normal (Gillespie-Gallery, Konstantakopoulou, Harlow, \& Barbur, 2013; Owsley, 2011).

De forma geral, mesmo apresentando distintos métodos para verificar como o contraste visual é alterado durante decorrer da idade, pode-se sugerir a existência de alterações no processo senso perceptivo visual ao longo da vida. Neste sentido, a função de SC pode ser utilizada como uma futura ferramenta prática para averiguar disfunções visuais associadas ao processo de envelhecimento.

Apesar destes achados, o presente trabalho demonstrou algumas limitações que podem ser sanadas em pesquisa futuras, a exemplo da quantidade reduzida de participantes, que foi apenas 18 por grupo. Outra carência metodológica está relacionada à amplitude de idade dos grupos. Pode-se dividir a amostra em diversos faixas etária (18-30, 31-40, 41-50, 51-60, e acima de 61 anos). Com isso, se permitirá determinar a partir de qual ponto começa a se registrar redução significativa da SC. A partir do momento em que os dados permitirem determinar em qual etapa do processo de desenvolvimento do sistema visual humano começa haver prejuízo na SC, estratégias compensatórios (DeLoss et al., 2015) podem começar a serem utilizadas para minimizar os efeitos negativos do envelhecimento. 


\section{Referências}

Allard, R., Renaud, J., Molinatti, S., \& Faubert, J. (2013). Contrast sensitivity, healthy aging and noise. Vision Research, 92, 47-52. http://dx.doi.org/10.1016/j.visres.2013.09.004

Andrade, D., Oliveira, M. J., Gadelha, M. J. N., Silva, J. A., Santos, D., \& Antonio, N. (2013). Visual processing of concentric sine-wave gratings in adolescents and adults. Avances en Psicología Latinoamericana, 31(1), 156-164. Retirado de http://www.scielo.org.co/scielo.php?script=sci_arttext\&pid=S1794-47242013000100013

Arundale, K. (1978). An investigation into the variation of human contrast sensitivity with age and ocular pathology. British Journal of Ophthalmology, 62(4), 213-215. http://dx.doi.org/10.1136/bjo.62.4.213

Bertolucci, P. H. F., Brucki, S. M. D., Campacci, S. R., \& Juliano, Y. (1994). The Mini-Mental State Examination in an outpatient population: influence of literacy. Arquivos de Neuro-Psiquiatria, 52(1), 01-07. http://dx.doi.org/10.1590/ S0004-282X1994000100001

Bishop, N. A., Lu, T., \& Yankner, B. A. (2010). Neural mechanisms of ageing and cognitive decline. Nature, 464(7288), 529-535. http://dx.doi.org/10.1038/nature08983

Birren, J. E. \& Schaie, K. W. (2006). Handbook of the psychology of aging (6”a ed.). San Diego, California, USA: Elsevier Academic Press.

Bissig, D. P., Roberts, R., \& Berkowitz, B. A. (2011). Vison Loss in Healthy Aging: Isolating the Contributions of Eye Optics, and Central Retinal Structure and Function. Investigative Ophthalmology \& Visual Science, 52(14), 1885-1885. Retirado de http://psycnet-apa-org.ez15.periodicos.capes.gov.br

Burton, K. B., Owsley, C., \& Sloane, M. E. (1993). Aging and neural spatial contrast sensitivity: photopic vision. Vision Research, 33(7), 939-946. http://dx.doi.org/10.1016/0042-6989(93)90077-A

Campbell, F. W. (1983). Why do we measure contrast sensitivity? Behavioural Brain Research, 10(1), 87-97. http:// dx.doi.org/10.1016/0166-4328(83)90154-7

Costa, T. L., Nogueira, R. M. T. B. L., Pereira, A. G. F., Mousinho, S. H. R., Marques, M. M., \& Santos, N. A. dos. (2009). Envelhecimento humano e sensibilidade ao contraste fotópica para frequências angulares. Psicologia: Teoria e Pesquisa, 25(4), 589-593. http://dx.doi.org/10.1590/S0102-37722009000400014

Costa, T. L., Nogueira, R. M. T. B. L., Pereira, A. G. F., Santos, N. A., Costa, T. L., Nogueira, R. M. T. B. L., ..., \& Santos, N. A. (2013). Differential effects of aging on spatial contrast sensitivity to linear and polar sine-wave gratings. Brazilian Journal of Medical and Biological Research, 46(10), 855-860. http://dx.doi.org/10.1590/1414-431X20133117

Cornsweet, T. N. (1970). Vision Perception. New York: Academic Press.

Crassini, B., Brown, B., \& Bowman, K. (1988). Age-related changes in contrast sensitivity in central and peripheral retina. Perception, 17(3), 315-332. http://dx.doi.org/10.1068/p170315

DeLoss, D. J., Watanabe, T., \& Andersen, G. J. (2015). Improving Vision Among Older Adults Behavioral Training to Improve Sight. Psychological Science. http://dx.doi.org/10.1177/0956797614567510

Derefeldt, G., Lennerstrand, G., \& Lundh, B. (1979). Age Variations in Normal Human Contrast Sensitivity. Acta Ophthalmologica, 57(4), 679-690. http://dx.doi.org/10.1111/j.1755-3768.1979.tb00517.x

Ellemberg, D., Lewis, T. L., Hong Liu, C., \& Maurer, D. (1999). Development of spatial and temporal vision during childhood. Vision Research, 39(14), 2325-2333. http://dx.doi.org/10.1016/S0042-6989(98)00280-6

Elliott, S. L. \& Werner, J. S. (2010). Age-related changes in contrast gain related to the M and P pathways. Journal of Vision, 10(4), 4.1-15. http://dx.doi.org/10.1167/10.4.4

Folstein, M. F., Folstein, S. E., \& McHugh, P. R. (1975). "Mini-mental state". A practical method for grading the cognitive state of patients for the clinician. Journal of Psychiatric Research, 12(3), 189-198. http://dx.doi.org/10.1016/00223956(75)90026-6

Gillespie-Gallery, H., Konstantakopoulou, E., Harlow, J. A., \& Barbur, J. L. (2013). Capturing age-related changes in functional contrast sensitivity with decreasing light levels in monocular and binocular vision. Investigative Ophthalmology \& Visual Science, 54(9), 6093-6103. http://dx.doi.org/10.1167/iovs.13-12119

Habak, C. \& Faubert, J. (2000). Larger effect of aging on the perception of higher-order stimuli. Vision Research, 40(8), 943-950. http://dx.doi.org/10.1016/S0042-6989(99)00235-7

Higgins, K. E., Jaffe, M. J., Caruso, R. C., \& deMonasterio, F. M. (1988). Spatial contrast sensitivity: effects of age, test?retest, and psychophysical method. Journal of the Optical Society of America A, 5(12), 2173-2180. http://dx.doi. org/10.1364/JOSAA.5.002173

Jia, W., Yan, F., Hou, F., Lu, Z. L., \& Huang, C. B. (2014). aCSF in Clinical Applications: Efficient Characterization and Classification of Contrast Sensitivity Funtions in Aging. Investigative Ophtalmology \& Visual Sience, 55(13), 762. Retirado de http://www.ncbi.nlm.nih.gov/pubmed

Kalia, A., Lesmes, L. A., Dorr, M., Bex, P. J., Gandhi, T., Chatterjee, G., \& Sinha, P. (2012). Measurements of contrast sensitivity functions Shefriow recovery from extended blindness. Perception, 154(41). ECVP: Abstract Supplement. http://dx.doi.org/10.1068/v120244

Lesmes, L. A., Wallis, J., Lu, Z.-L., Jackson, M. L., \& Bex, P. (2012). Clinical Application Of A Novel Contrast Sensitivity Test To A Low Vision Population: The Quick CSF Method. Investigative Ophthalmology \& Visual Science, 53(14), 4358-4358. Retirado de http://psycnet-apa-org.ez15.periodicos.capes.gov.br

Lesmes, L. A., Jackson, M., Wallis, J., \& Bex, P. J. (2013). The reliability of the quick CSF method for contrast sensitivity assessment in low vision. Investigative Ophthalmology \& Visual Science, 54, 2762. Retirado de http://psycnet-apa-org. ez15.periodicos.capes.gov.br 
Li, K. Z. H. \& Lindenberger, U. (2002). Relations between aging sensory/sensorimotor and cognitive functions. Neuroscience and Biobehavioral Reviews, 26(7), 777-783. http://dx.doi.org/10.1016/S0149-7634(02)00073-8

Mateus, C., Lemos, R., Silva, M. F., Reis, A., Fonseca, P., Oliveiros, B., \& Castelo-Branco, M. (2013). Aging of Low and High Level Vision: From Chromatic and Achromatic Contrast Sensitivity to Local and 3D Object Motion Perception. PLoS ONE, 8(1). http://dx.doi.org/10.1371/journal.pone.0055348

Malloy-Diniz, L. F., Fuentes, D., \& Cosenza, R. M. (2013). Neuropsicologia do Envelhecimento: Uma abordagem multidimensional. Porto Alegre: Artmed.

McGrath, C. \& Morrison, J. D. (1981). The Effects of Age on Spatial Frequency Perception in Human Subjects. Quarterly Journal of Experimental Physiology, 66(3), 253-261. http://dx.doi.org/10.1113/expphysiol.1981.sp002554

Owsley, C. (2011). Aging and vision. Vision Research, 51(13), 1610-1622. http://dx.doi.org/10.1016/j.visres.2010.10.020

Owsley, C., Sekuler, R., \& Siemsen, D. (1983). Contrast sensitivity throughout adulthood. Vision Research, 23(7), 689-699. http://dx.doi.org/10.1016/0042-6989(83)90210-9

Park, D. C., Polk, T. A., Park, R., Minear, M., Savage, A., \& Smith, M. R. (2004). Aging reduces neural specialization in ventral visual cortex. Proceedings of the National Academy of Sciences of the United States of America, 101(35), 13091-13095. http://dx.doi.org/10.1073/pnas.0405148101

Pelli, D. G. \& Bex, P. (2013). Measuring contrast sensitivity. Vision Reserarch, 20(90), 10-14. http://dx.doi.org/10.1016/j. visres.2013.04.015

Roorda, A. (2011). Adaptive optics for studying visual function: a comprehensive review. Journal of Vision, 11(7). http:// dx.doi.org/10.1167/11.5.6

Rosen, R., Jayaraj, J., Bharadwaj, S. R., Weeber, H. A., Mooren, M. V. der, \& Piers, P. A. (2015). Contrast sensitivity in patients with macular degeneration. Investigative Ophthalmology \& Visual Science, 56(7), 2224-2224. Retirado de http://psycnet-apa-org.ez15.periodicos.capes.gov.br

Salthouse, T. A. \& Czaja, S. J. (2000). Structural constraints on process explanations in cognitive aging. Psychology and Aging, 15(1), 44-55. http://dx.doi.org/10.1037/0882-7974.15.1.44

Santos, N. A. dos \& França, V. de C. R. de M. (2008). Contrast sensitivity to sine-wave gratings of low spatial frequencies in children. Estudos de Psicologia (Campinas), 25(2), 177--184. http://dx.doi.org/10.1590/S0103-166X2008000200002

Santos, N. A. dos, Oliveira, A. B., Nogueira, R. M. T. B. L., Cruz, É. D. N. da, \& Simas, M. L. de B. (2006). Os efeitos da idade na percepção visual de grades senoidais em luminância baixa. Psicologia: Reflexão e Crítica, 19(3), 415-421. http://dx.doi.org/10.1590/S0102-79722006000300010

Santos, N. A. dos, Simas, M. L. de B., \& Nogueira, R. M. T. B. L. (2003). Processamento visual da forma em idosos: curvas de limiar de contraste para freqüências angulares e senoidais. Psicol. reflex. crit, 16(2), 271-277. http://dx.doi. org/10.1590/S0102-79722003000200007

Schefrin, B. E., Tregear, S. J., Harvey Jr, L. O., \& Werner, J. S. (1999). Senescent changes in scotopic contrast sensitivity. Vision Research, 39(22), 3728-3736. http://dx.doi.org/10.1016/S0042-6989(99)00072-3

Scialfa, C. T., Kline, D. W., \& Wood, P. K. (2002). Structural modeling of contrast sensitivity in adulthood. Journal of the Optical Society of America A, 19(1), 158-165. http://dx.doi.org/10.1364/JOSAA.19.000158

Sekuler, R. \& Hutman, L. P. (1980). Spatial Vision and Aging. I: Contrast Sensitivity. Journal of Gerontology, 35(5), 692-699. http://dx.doi.org/10.1093/geronj/35.5.692

Silva, M. F., d'Almeida, O. C., Oliveiros, B., Mateus, C., \& Castelo-Branco, M. (2014). Development and aging of visual hemifield asymmetries in contrast sensitivity. Journal of Vision, 14(12). http://dx.doi.org/10.1167/14.12.19

Skalka, H. W. (1980). Effect of age on Arden grating acuity. British Journal of Ophthalmology, 64(1), 21-23. http:// dx.doi.org/10.1136/bjo.64.1.21

Tang, Y. \& Zhou, Y. (2009). Age-related decline of contrast sensitivity for second-order stimuli: Earlier onset, but slower progression, than for first-order stimuli. Journal of Vision, 9(7), 18. http://dx.doi.org/10.1167/9.7.18

Wetherill, G. B. \& Levitt, H. (1965). Sequential Estimation of Points on a Psychometric Function. British Journal of Mathematical and Statistical Psychology, 18(1), 1-10. http://dx.doi.org/10.1111/j.2044-8317.1965.tb00689.x

\footnotetext{
Autores:

Maria José Nunes Gadelha - Doutoranda, Universidade Federal da Paraíba.

Joenilton Saturnino Cazé da Silva - Mestrando, Universidade Federal da Paraíba.

Jandilson Avelino da Silva - Doutor, Universidade Federal da Paraíba.

Michael Jackson de Oliveira Andrade - Doutorando, Universidade Federal da Paraíba.

Débora Najda de Medeiros Viana - Mestre, Universidade Federal da Paraíba.

Natanael Antonio dos Santos - Doutor, Universidade Federal da Paraíba.

Endereço para correspondência:

Maria José Nunes Gadelha

Rua Enfermeira Ana Maria Barbosa de Almeida, 631

João Pessoa, PB, Brasil

<nunesgadelha@hotmail.com>

Recebido em: 19.11 .15

Aceito em: 29.05.16
} 